\title{
Evaluation of Treatment Response in Lupus Nephritis
}

\author{
Anca BOBIRCA ${ }^{1,2}$, Florin BOBIRCA², Anca FLORESCU' ${ }^{1}$, Carmen IORGUS'ㄱ, Radu TINCA², \\ Anca MUSETESCU ${ }^{3}$, Mihai BOJINCA ${ }^{1,2}$, loan ANCUTA ${ }^{1,2}$
}

\begin{abstract}
Objectives: To evaluate the effectiveness of the treatment reflected by the rate of response to therapy at 6 months and 12 months of follow-up respectively. Methods: We retrospectively analyzed clinical, laboratory data, treatment regimens, the type of response and relapse rate of 51 patients diagnosed with LN between January 2017 and February 2020. Results:47.06\% of the patients underwent renal biopsy, classes III and IV being the most common lupus nephritis types (totaling 35.3\% of biopsied patients). All induction therapy choices analyzed in the study- CYC, Glucocorticoids (GCs) and MMF- proved effective at reducing the proteinuria of the patients $(p=0.001, p=0.012$ and $p=0,019$ respectively. The 12 months evaluation demonstrated an ascending trend of the complete response, starting from $27.45 \%$ at 6 months and almost doubling at 1 year (56.86\%). Almost half of patients (49.02\%) did not relapse, while most of them (27.45\%) had only 1 relapse. Analyzing the risk of relapse for each induction drug used, CYC had the highest rate of recurrence (62.07\%). The use of MMF as a maintenance drug associated the lowest degree of recurrence. Conclusions: Both $\mathrm{CYC}$ and MMF as induction therapy are significantly effective in reducing proteinuria. The complete response was more frequently identified as an endpoint at 12 months of follow-up.
\end{abstract}

Keywords: lupus nephritis, treatment, response relapse.

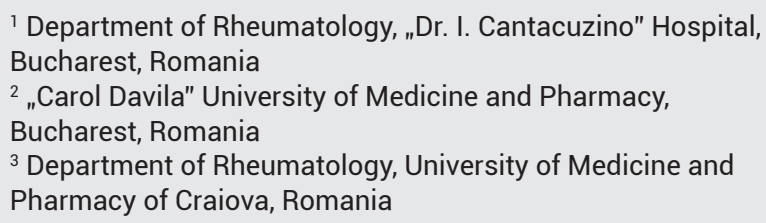

Corresponding author:

Florin-Teodor BOBIRCA, "Carol Davila" University of Medicine and Pharmacy, Bucharest, Romania.

E-mail: dr.bobirca@gmail.com 


\section{INTRODUCTION}

Systemic lupus erythematosus (SLE) is a complex, multisystemic autoimmune disease with a wide range of clinical manifestations. Lupus nephritis (LN) is an important organ involvement in terms of survival rate, being one of the main causes of morbidity and mortality during the evolution of SLE.

Based on microscopy, immunofluorescence and electronic microscopy changes, International Society of Nephrology/Renal Pathology Society (ISF/ISN) and the World Health Organization (WHO) have recommended the classification of lupus nephritis into 6 classes as follows:

- Class I: minimal mesangial lupus nephritis; glomeruli appear normal on light microscopy. Immunofluorescence shows immune complex deposits in the mesangial space.

- Class II: proliferative mesangial LN; mesangial proliferation is seen on light microscopy. Immunofluorescence shows immune complex deposits in the mesangial space.

- Class III: focal lupus nephritis. Immune complex deposits may be visualized in the mesangial, subendothelial and/or subepithelial space on immunofluorescence imaging.

- Class IV: diffuse LN; immune complex deposits may occur in the mesangial, subendothelial and/ or the subepithelial space. Lesions may be segmental, involving less than $50 \%$ of the glomeruli, or global, that involves more than 50\%.

- Class V: membranous LN; immune complex deposits are in the mesangial and subepithelial space. Capillary loops are thickened due to subepithelial immune complex deposits. At this class, nephrotic range proteinuria occurs. Class $\mathrm{V}$ may also include Class III and IV pathology.

- Class VI: advanced sclerosing LN; most of the glomeruli are sclerosed. However, immune complex deposits are not visualized on immunofluorescence since more than $90 \%$ of the glomeruli are scarred ${ }^{1}$.

Nephritis occurs in about $50 \%$ of people with SLE and the prognosis is strongly influenced by the early initiation of treatment. This makes clinicians' vigilance essential for a better prognosis and longer life expectancy of patients with SLE.

The aim of the therapy in lupus nephritis is to use immunosuppressive drugs to improve renal outcome and, consequently, the patient's survival rate.
The immunosuppressive therapy has two phases: induction and maintenance.

Induction therapy regimens:

a) The National Institutes of Health (NIH) regimen of CYC $0,75-1 \mathrm{~g} / \mathrm{m} 2$ body surface monthly for 6 months together with Methyl prednisone intravenous (IV) and oral $\mathrm{GCs}^{2}$;

b) The Euro-Lupus regimen of CYC administered in 6 rounds of $500 \mathrm{mg}$ once every 2 weeks combined with methyl prednisone pulse therapy 3 daily doses of $750 \mathrm{mg}$ IV plus oral GCs $0,5 \mathrm{mg} / \mathrm{kg} / \mathrm{day}^{3}$;

c) MMF regimen of 2-3g daily for 6 months ${ }^{4}$.

The Euro-Lupus Trial showed similar efficacy between a) and b); the patients were Caucasian descent and the level of nephritis intensity was moderate ${ }^{3}$.

Maintenance therapy regimens:

a) Azathioprine (AZA) $2 \mathrm{mg} / \mathrm{kg} /$ day with a maximum dose of $150 / 200 \mathrm{mg} /$ day $^{5}$;

b) MMF 2x1000mg doses daily;

c) CYC (no longer recommended as maintenance therapy as it has a lower efficacy as well as more side effects $)^{6}$.

All regimens are generally used, the choice having to be made by the clinician taking into account the particularities of the patient.

Active urinary sediment is associated with periods of disease activity. It is defined as the presence of $>5$ red blood cells or $>5$ white blood cells per high-power field and/or the presence of cellular casts ${ }^{8}$.

The most frequent pathological changes of the urinary sediment found in $\mathrm{LN}$, in descending order are leukocyturia, hematuria, granular casts and hyaline casts 9 .

We defined the complete response (CR) as patients with serum creatinine $<1.2 \mathrm{mg} / \mathrm{dL}$, decrease to the initial values or $\pm 15 \%$ of baseline value in patients with creatinine $\geq 1.2 \mathrm{mg} / \mathrm{dl}$, proteinuria $\leq 0.5 \mathrm{~g} / 24 \mathrm{~h}$, inactive urinary sediment ( $\leq 5$ red blood cells, $\leq 5$ leukocytes and no red blood cell casts) and serum albumin $>3 \mathrm{~g} / \mathrm{dl}$. Partial response (PR) was defined in patients with nephrotic proteinuria as decreased proteinuria $<3.5 \mathrm{~g} / 24 \mathrm{~h}$, in patients with proteinuria $<3.5 \mathrm{mg} / 24 \mathrm{~h}$ a $>50 \%$ reduction in proteinuria in both situations, stabilization $( \pm 25 \%)$ and improvement in serum creatinine ${ }^{7}$. 


\section{MATERIALS AND METHODS}

51 patients were included in this cross sectional, observational, retrospective study. They were diagnosed with SLE and LN based on the ACR/EULAR 2019 classification criteria and the ISF/ISN histopathological classification of $\mathrm{LN}$ respectively.

The mandatory inclusion criteria was the presence of renal involvement defined as increased proteinuria, the presence of active urinary sediment, positive biopsy for renal impairment or an increased serum creatinine level.

The main objective of this study was to evaluate the effectiveness of the treatment reflected by the rate of response to therapy at 6 months and 12 months of follow-up respectively, and the number of relapses. These target both the entire study group and the subgroup of patients who underwent renal biopsy $(n=24)$.

The data was collected from the hospital's paperwork of all the patients admitted to the Internal Medicine and Rheumatology Clinic of „Dr I. Cantacuzino” Clinical Hospital, Bucharest, between 10.01.201720.02.2020. The patient's data was maintained confidential according to the Helsinki Declaration. The study protocol was approved by the ethics committee of „Dr. I. Cantacuzino” Hospital, Bucharest. Written informed consent was obtained from each patient. The study was conducted in accordance with the principles of the Declaration of Helsinki.

For the initial characterization of the study group, we gathered information regarding the current age, age at diagnosis, gender, duration of the disease, duration between diagnosis and onset of renal impairment, smoking status, the histopathological class of lupus nephritis and the therapeutic regimens of induction and maintenance, respectively.

Efficacy indicators in our study were considered complete response $(\mathrm{CR})$, partial response $(\mathrm{PR})$, ther- apeutic resistance/no response and number of relapses, respectively. In this regard, we analyzed the following parameters: $24 \mathrm{~h}$ proteinuria, serum creatinine level, estimated glomerular filtration rate (eGFR), urinary sediment. All this were performed at diagnosis and at the 6 and 12-months follow-ups.

Data was analyzed using IBM SPSS Statistics (Statistical Package for the Social Sciences) and $p$ value $<0.05$ was considered statistically significant.

\section{RESULTS}

Out of the 51 patients included in this study, 50 (98.04\%) were female and $1(1.96 \%)$ patient was male. Regarding the smoking status, only $15.69 \%$ were smokers. The mean age of the patients was 43 years, ranging from 20 to 75 years. The mean age at diagnosis was 28 years, with limits between 14 and 60, with an average disease duration of 14 years.

The mean duration between diagnosis and renal impairment was 5 years. We considered this value the boundary between early and late onset of the renal involvement.

Mean proteinuria level at diagnosis in the study group was $3.45 \mathrm{~g} / 24 \mathrm{~h}$. Using this parameter, 50.98\% of patients had nephrotic syndrome (proteinuria $\geq 3.5 \mathrm{~g} / 24 \mathrm{~h}$ ), and the remaining $49.02 \%$ had nephritic syndrome (proteinuria $<3.5 \mathrm{~g} / 24 \mathrm{~h}$ ). The mean creatinine level at diagnosis was $0.90 \mathrm{mg} / \mathrm{dl}$, ranging from 0.46 to $2 \mathrm{mg} / \mathrm{dl}$. The mean eGFR level was 94.16 , with limits between 31.96 and $184.1 \mathrm{ml} / \mathrm{min} / 1.73 \mathrm{~m} 2$.

$88.24 \%$ of the patients had urinary active sediment at diagnosis of renal manifestation.

Patients with early onset of LN showed a higher proteinuria than the patients with late onset of $\mathrm{LN}$ ( $4.17 \mathrm{~g} / 24 \mathrm{~h}$ versus $2.13 \mathrm{~g} / 24 \mathrm{~h}, \mathrm{p}=0.072)$.

Out of all patients, $52.94 \%$ of them did not have a renal biopsy performed.

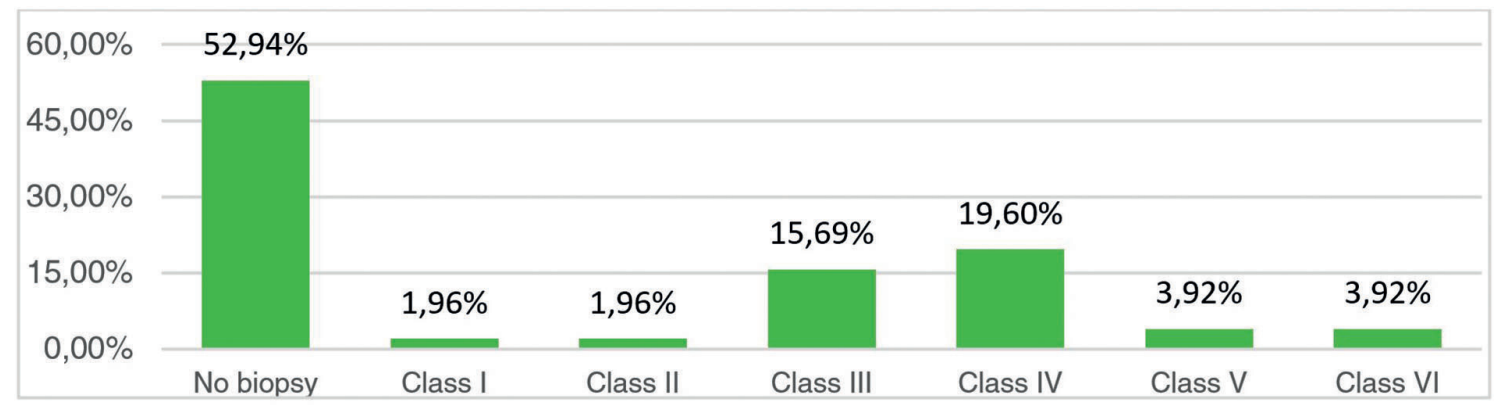

Figure 1. Classification of patients based on the biopsy status and histopathological result 
Regarding the biopsy results, most patients belonged to the lupus nephritis class IV (19.61\%), followed, in order, by class III (15.69\%), class V and VI with a percentage of $3.92 \%$ for each category. Classes I and II were the least common types of lupus nephritis with a percentage of $1.92 \%$ each.

Table 1. Distribution of Induction and Maintenance therapies in the entire study group

\begin{tabular}{|l|l|l|l|}
\hline Induction therapy & Patients $(\mathbf{n}, \mathbf{p} \%)$ & Maintenance therapy & Patients (n, \%) \\
\hline CYC iv & $29,56.86 \%$ & CYC oral & $10,19.61 \%$ \\
\hline MMF & $6,11.76 \%$ & MMF & $8,15.69 \%$ \\
\hline Steroids iv & $8,15.69 \%$ & Steroids oral & $49,96.08 \%$ \\
\hline None & $8,15.69 \%$ & AZA & $27,52.94 \%$ \\
\hline & - & HCQ & $43,84.31 \%$ \\
\hline & - & Other (CYA, TAC, MTX) & $5,9.8 \%$ \\
\hline
\end{tabular}

CYC-Cyclophosphamide, MMF-Mycophenolate Mofetil, AZA-Azathioprine,

HCQ-Hydroxychloroquine, CYA-Cyclosporine A, TAC-Tacrolimus, MTX-Methotrexate

Studying the induction therapy in the entire group, CYC iv is the most frequent immunosuppressive used, $56,86 \%$ of the patients having received it under both Euro-Lupus and NYH regime, followed by pulse steroid therapy in $15.69 \%$ of cases.

Regarding the maintenance therapy in the entire study group, oral steroids were used in almost all patients (96.08\%), followed, in order of frequency, by HCQ (84.31\%), AZA (52.94\%), in contrast to MMF that was used in only $15.69 \%$ of cases.
Extensive analysis of the biopsied group revealed that Cyclophosphamide was the most commonly used agent in lupus nephritis of classes III and IV, followed by Mycophenolate Mofetil.

The most sustained treatment in the maintenance phase targeted classes III and IV of lupus nephritis. The combined therapy including steroids, $\mathrm{HCQ}$ and AZA was the most common maintenance regimen used. MMF was recommended in only 5 patients, while oral CYC was preferred in 4 cases.

The main objective of this study was to evaluate the effectiveness of the treatment reflected by the rate of response to therapy at 6 months and 12 months of follow-up respectively, and the number of relapses.

Table 2. Distribution of Induction and Maintenance therapies in the biopsied group

\begin{tabular}{|c|c|c|c|c|c|c|c|c|c|c|}
\hline \multirow[b]{2}{*}{$\begin{array}{l}\text { Class of lupus } \\
\text { nephritis; no of } \\
\text { patients }\end{array}$} & \multicolumn{4}{|c|}{ Induction therapy } & \multicolumn{6}{|c|}{ Maintenance therapy } \\
\hline & Steroids iv & CYC iv & MMF & None & CYC oral & MMF & Steroids oral & AZA & HCQ & $\begin{array}{l}\text { Other } \\
\text { CYA } \\
\text { TAC } \\
\text { MTX }\end{array}$ \\
\hline I (1) & $\begin{array}{l}1 \\
100 \%\end{array}$ & - & - & - & - & - & $\begin{array}{l}1 \\
100 \%\end{array}$ & - & - & $1100 \%$ \\
\hline II (1) & $\begin{array}{l}1 \\
100 \%\end{array}$ & - & - & - & - & - & $\begin{array}{l}1 \\
100 \%\end{array}$ & - & $1100 \%$ & - \\
\hline III (8) & $112,5 \%$ & $562.5 \%$ & $225 \%$ & - & $112.5 \%$ & $225 \%$ & $\begin{array}{l}7 \\
87.5 \%\end{array}$ & $8100 \%$ & $787.5 \%$ & $112.5 \%$ \\
\hline IV (10) & $\begin{array}{l}1 \\
10 \%\end{array}$ & \begin{tabular}{|l}
6 \\
$60 \%$
\end{tabular} & $220 \%$ & $110 \%$ & $\begin{array}{l}3 \\
30 \%\end{array}$ & $330 \%$ & $10100 \%$ & $6100 \%$ & $\begin{array}{l}8 \\
80 \%\end{array}$ & $\begin{array}{l}3 \\
30 \%\end{array}$ \\
\hline $\mathrm{V}(2)$ & - & $2100 \%$ & - & - & $150 \%$ & - & $\begin{array}{l}2 \\
100 \%\end{array}$ & - & $\begin{array}{l}2 \\
100 \%\end{array}$ & - \\
\hline VI (2) & - & $2100 \%$ & - & - & - & - & $\begin{array}{l}2 \\
100 \%\end{array}$ & $150 \%$ & - & - \\
\hline
\end{tabular}




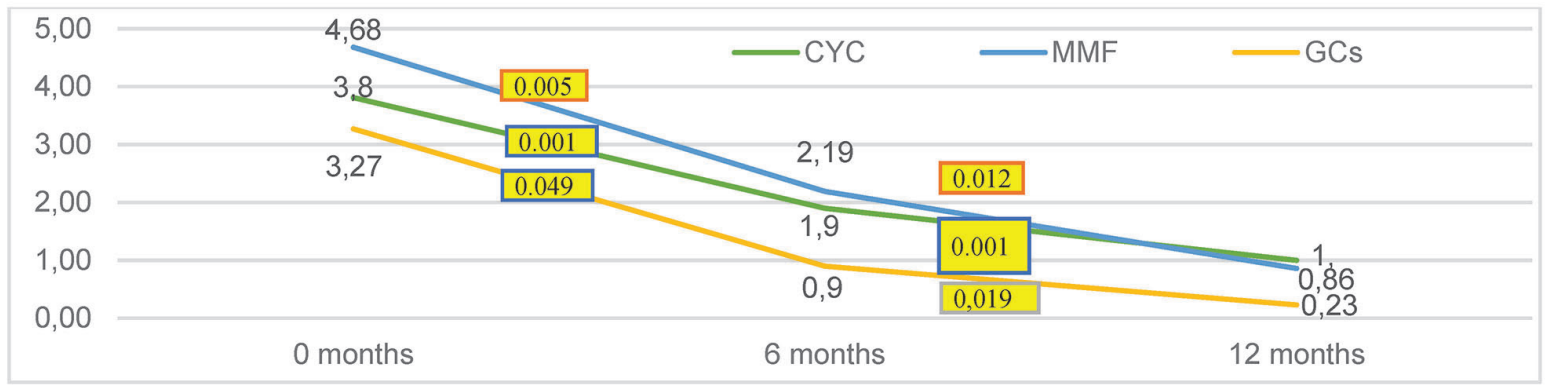

Figure 2. Variation of proteinuria under the induction scheme

Patients in all three regimens of therapeutic induction showed a decreasing trend in mean proteinuria. All variations at both 6 and 12 months were observed to be statistically significant (the $\mathrm{p}$ value calculated using the $T$ test with a statistical significance set at $\mathrm{p}<0.05$ ).

Regarding the laboratory tests at the 12 months follow-up, we noticed that there was no patient with nephrotic syndrome after 1 year, the mean proteinuria being $0.73 \mathrm{~g} / 24 \mathrm{~h}$ with limits between 0 and 3.2 $\mathrm{g} / 24 \mathrm{~h}$. The mean creatinine and mean eGFR remained constant at the endpoint $(0.8 \mathrm{mg} / \mathrm{dl}$ and $97.94 \mathrm{ml} /$ $\mathrm{min} / 1.73 \mathrm{~m} 2$, respectively).

The 12 months evaluation demonstrated an ascending trend of the complete response, starting from $27.45 \%$ at 6 months and almost doubling at 1 year (56.86\%). The therapeutic resistance percentage remained stable (3.92\%) between the 2 medical evaluations.



Figure 3. Response at 6 and 12 months after initiation of treatment in the entire study group

Almost half of patients (49.02\%) did not relapse, while most of them $(27.45 \%)$ had only 1 relapse. The percentage of patients who had 2 relapses was $11.76 \%$ and at the same time $9.8 \%$ of them developed more than 2 flares.

Table 3. Response rate and number of relapses in the biopsied group

\begin{tabular}{|c|c|c|c|c|c|c|c|c|c|c|c|}
\hline \multirow{3}{*}{$\begin{array}{l}\text { Class of lupus } \\
\text { nephritis }\end{array}$} & \multirow{3}{*}{ Pts $(\mathrm{n}=25)$} & \multicolumn{6}{|c|}{ Response } & \multirow{2}{*}{\multicolumn{4}{|c|}{ Number of relapses }} \\
\hline & & \multicolumn{3}{|c|}{ Response at 6 months } & \multicolumn{3}{|c|}{ Response at 12 months } & & & & \\
\hline & & PR & CR & No response & PR & CR & No response & 0 & 1 & 2 & $\geq 3$ \\
\hline I & 1 & & & $1(100 \%)$ & & & $1(100 \%)$ & & 1 & & \\
\hline II & 1 & $1(100 \%)$ & & & & $1(100 \%)$ & & 1 & & & \\
\hline III & 8 & $7(87.5 \%)$ & $1(12,5 \%)$ & & $4(50 \%)$ & $\begin{array}{l}4 \\
(50 \%)\end{array}$ & & 4 & 1 & 2 & 1 \\
\hline IV & 10 & $8(80 \%)$ & $2(20 \%)$ & & $5(60 \%)$ & $\begin{array}{l}4 \\
(40 \%)\end{array}$ & & 4 & 2 & 2 & 2 \\
\hline $\mathrm{v}$ & 2 & $1(50 \%)$ & $1(50 \%)$ & & $1(50 \%)$ & $\begin{array}{l}1 \\
(50 \%)\end{array}$ & & 1 & & & 1 \\
\hline VI & 2 & $1(50 \%)$ & & $1(50 \%)$ & $1(50 \%)$ & & $1(50 \%)$ & $2^{*}$ & & & \\
\hline
\end{tabular}

*End stage renal disease 
Particularly for the biopsied population, the only two patients that showed no response at the 6 and 12 months follow-ups belonged to the first class of lupus nephritis and sixth class respectively.

Surprisingly, the most aggressive classes of lupus nephritis (III, IV) did not show therapeutic resistance.

Most patients had no relapse after the induction therapy. Relapses occurred mostly in classes III and IV of lupus nephritis. The only patient with class I lupus nephritis had 1 relapse, while one patient from the class $V$ category had more than 3 relapses.

Among the patients evaluated, smokers were prone to relapse (OR=0.522, 95\% CI: $0.111-2.462)$, a result that was not statistically validated.

Analyzing the risk of relapse for each induction drug used, it was concluded that $62.07 \%$ of the patients under CYC had at least one relapse, $16.67 \%$ of the patients under MMF had one relapse, $50 \%$ of patients with GCs as induction had at least one relapse and $37.50 \%$ of those without induction therapy had also, at least one relapse.

Regarding maintenance therapy both AZA and MMF had similar relapse rates (48.15\% versus $50 \%$ ), while CYC had a higher rate than both of them (70\%). The average relapse rate for each of the maintenance drugs is 0.875 on MMF, 0,89 relapses on AZA while CYC had 1,7 relapses.

Analyzing the relapse rates, based on the period between the diagnosis of the disease and the onset of renal involvement, we observed that $54.55 \%$ of patients with early onset of LN (18 patients out of 33) developed at least 1 relapse, while only $44.44 \%$ (8 of 18) patients with late onset of $\mathrm{LN}$ had at least 1 relapse. No post-relapse re-biopsies were performed.

In addition, none of the patients included in the study received monoclonal antibodies in the therapeutic regimes. In this regard, we suggest the need for a more extensive analysis, ideally in a prospective method performed on a larger number of patients.

\section{CONCLUSIONS}

In conclusion, the study shows that early identification and treatment of renal involvement in patients with SLE are associated with a better prognosis, a significant decrease in proteinuria, and a complete or partial therapeutic response.

Both CYC and MMF as induction therapy are significantly effective in reducing proteinuria $(p=0.001$ and $\mathrm{p}=0.012$, respectively), the appropriate drug being the choice of the clinician based on the patient's particularities.

The complete response was more frequently identified as an endpoint at 12 months of follow-up. This in accordance with the latest EULAR recommendations which extended the definition of therapeutic resistance from 6 months to 12 months after treatment 10,11. The use of Mycophenolate Mofetil as a maintenance drug associated the lowest degree of recurrence, while smoking was the most common risk factor for relapse.

The most aggressive classes of lupus nephritis (III, IV) did not show therapeutic resistance after 12 months of follow-up, with CYC being the most used induction agent.

None of the patients included in this study showed nephrotic syndrome after 1 year of therapy, the mean proteinuria being $0.73 \mathrm{~g} / 24 \mathrm{~h}$ with limits between 0 and $3.2 \mathrm{~g} / 24 \mathrm{~h}$.

Patients with early onset of LN had higher proteinuria levels at diagnosis, in comparison to the patients with late onset of $\mathrm{LN}(4.17 \mathrm{~g} / 24 \mathrm{~h}$ versus $2.13 \mathrm{~g} / 24 \mathrm{~h}$, $\mathrm{p}=0.072$ ). In the same time, they showed a better response to treatment with a more accelerated decrease of proteinuria at the 12 months endpoint. Analyzing a similar study, conducted by Delfino J et al, that included 71 patients, no differences were observed between the two groups in terms of proteinuria or response rate to therapy ${ }^{12}$.

Analyzing the risk of relapse for each induction drug used, CYC had the highest rate of recurrence (62.07\%), in opposition to the result obtained by Jasvinder A. Singh et al, according to which immunosuppressive drugs (including CYC) were superior to steroids. This can be explained by the fact that, in our study, corticosteroids were used in only $15.69 \%$ of cases (vs. $56.86 \%$ for $\mathrm{CYC})^{13}$.

Regarding maintenance therapy both AZA and MMF had similar relapse rates $(48.15 \%$ versus $50 \%)$, while CYC had a higher rate than both of them (70\%). Several studies observed that MMF is better than AZA in terms of renal relapse ${ }^{13,14}$.

Re-biopsy of therapeutic-resistant patients would be necessary for targeted and a more effective therapy. A possible solution for these patients could be biological therapy, which was not used in this study.

Extending the analysis over a longer period of time and to a larger group of patients would be useful to identify the particularities of the response rate for each of the therapeutic regimens. 
Compliance with ethics requirements: The authors declare no conflict of interest regarding this article. The authors declare that all the procedures and experiments of this study respect the ethical standards in the Helsinki Declaration of 1975, as revised in 2008(5) and the national law. Informed consent was obtained from the patient described in the clinical case and his parents.

\section{References}

1. Musa R, Brent LH, Qurie A. Lupus Nephritis. [Updated 2020 Aug 15]. In: StatPearls [Internet]. Treasure Island (FL): StatPearls Publishing; 2021 Jan https://www.ncbi.nlm.nih.gov/books/ NBK499817/

2. Illei GG, Austin HA, Crane M, et al. Combination therapy with pulse cyclophosphamide plus pulse methylprednisolone improves long-term renal outcome without adding toxicity in patients with lupus nephritis. Ann Intern Med. 2001 Aug 21;135(4):248-57.

3. Houssiau FA, Vasconcelos C, D'Cruz D, et al. Immunosuppressive therapy in lupus nephritis: the Euro-Lupus Nephritis Trial, a randomized trial of low-dose versus high-dose intravenous cyclophosphamide. Arthritis Rheum. 2002 Aug;46(8):2121-31.

4. Appel GB, Contreras G, Dooley MA, et al. Aspreva Lupus Management Study Group. Mycophenolate mofetil versus cyclophosphamide for induction treatment of lupus nephritis. J Am Soc Nephrol. 2009 May;20(5):1103-12.

5. Falk R.J., MD, Dall'Era M., MD, Appel G.B., MD - Treatment and prognosis of diffuse or focal proliferative lupus nephritis ed. UpToDate 2019. Waltham, MA: UpToDate; https://www.uptodate.com/contents/treatment-and-prognosis-of-diffuse-or-focal-proliferative-lupus-nephritis

6. Boumpas DT, Austin HA 3rd, Vaughan EM, Yarboro CH, Klippel JH, Balow JE. Risk for sustained amenorrhea in patients with systemic lupus erythematosus receiving intermittent pulse cyclophosphamide therapy. Ann Intern Med. 1993 Sep 1;119(5):366-9.

7. Guillermo Ruiz Irastorzaa, Gerard Espinosab, Miguel A. Frutosc, et al. Diagnosis and treatment of lupus nephritis. Consensus document from the systemic auto-immune disease group (GEAS) of the Spanish Society of Internal Medicine (SEMI) and the Spanish Society of Nephrology (S.E.N.). Nefrologia 2012;32(Suppl.1)1-35
8. Liang, M.H., Schur, P.H., Fortin, P.,et al. The American college of rheumatology response criteria for proliferative and membranous renal disease in systemic lupus erythematosus clinical trials. Arthritis \& Rheumatism, 54: 421-432. https://doi.org/10.1002/ art. 21625

9. Saxena R, Mahajan T, Mohan C. Lupus nephritis: current update Arthritis Res Ther. 2011;13(5):240. doi:10.1186/ar3378

10. Dall'Era M, Cisternas MG, Smilek DE, et al. Predictors of longterm renal outcome in lupus nephritis trials: lessons learned from the Euro-Lupus Nephritis cohort. Arthritis Rheumatol. 2015 May;67(5):1305-13.

11. Tamirou F, Lauwerys BR, Dall'Era M, et al. MAINTAIN Nephritis Trial Investigators. A proteinuria cut-off level of $0.7 \mathrm{~g} /$ day after 12 months of treatment best predicts long-term renal outcome in lupus nephritis: data from the MAINTAIN Nephritis Trial. Lupus Sci Med. 2015 Nov 12;2(1):e000123.

12. Delfino, J., dos Santos, T.A.F.G. \& Skare, T.L. Comparison of lupus patients with early and late onset nephritis: a study in 71 patients from a single referral center. Adv Rheumatol 60, 5 (2020). https:// doi.org/10.1186/s42358-019-0105-5

13. Jasvinder A. Singh, Alomgir Hossain, Ahmed Kotb, et al.. Treatments for Lupus Nephritis: A Systematic Review and Network Metaanalysis. The Journal of Rheumatology Oct 2016, 43 (10) 1801-1815; DOI: 10.3899/jrheum.160041

14. Chen, Y., Sun, J., Zou, K., Yang, Y., \& Liu, G. (2017). Treatment for lupus nephritis: an overview of systematic reviews and meta-analyses. Rheumatology International, 37(7), 1089-1099. doi:10.1007/ s00296-017-3733-2 